\title{
Monitoría Fetal Anteparto en la Unidad de Alto Riesgo del Hospital San Ignacio
}

\author{
Dres. Jorge E. Medina Murillo* y Jaime Bechara Chamat**
}

Se agradece la colaboración prestada por el Dr. Mario Garcés Ferrer, Jefe de Estadística del Hospital San Ignacio.

\section{INTRODUCCION}

Con la implementación del concepto del Alto Riesgo en la práctica obstétrica, se ha hecho indispensable la evaluación sistemática del medio ambiente fetal.

Entre los métodos más confiables y de fácil aplicación se encuentra el empleo de la tococardiografía o monitoria fetal, la cual en un comienzo se utilizó durante el trabajo de parto, pero en la última década se ha conocido mejor en base a los trabajos de Hammacher (1966) (1). Pose y colaboradores (1966) (1), Ray y colaboradores (1972) (8), y Schifrin (10), quienes han demostrado la bondad de estos procedimientos.

Hoy día es aceptado que la prueba sin stress (NST) es un buen índice de predicción de bienestar fetal cuando es reactiva; cuando no lo es, debe ser complementada con una prueba de tolerancia

Profesor Titular de Obstetricia y Ginecología.

** Residente III. a las contracciones (PTC, Prueba de Pose - Prueba de Tolerancia a la Oxitocina) (2), (3), (9), (8), (10).

El objeto de este trabajo es presentar los resultados de nuestra experiencia en la Unidad de Alto Riesgo Obstétrico del Hospital Universitario de San Ignacio con estas pruebas.

\section{Material y Métodos}

Este estudio representa el análisis de las pruebas de monitoría fetal anteparto realizadas en la Unidad de alto riesgo del Departamento de Obstetricia del Hospital Universitario San Ignacio, durante un período comprendido entre el mes de agosto de 1980 y el mes de junio de 1981.

Los requisitos para ser incluidas en este estudio fueron los siguientes:

1. Pacientes que estuvieran en control prenatal en la Consulta de Alto Riesgo.

2. Pacientes que estuvieron hospitalizadas en la Unidad de Alto Riesgo y 
tuvieran parto en el Hospital San Ignacio.

162 pacientes cumplieron estos requisitos y constituyen el universo del estudio.

Para las pruebas se utilizó un Monitor Fetal Corometrics, Modelo $111 \mathrm{~A}$.

Utilizando transductor ultrasónico para el registro de la frecuencia cardíaca fetal (FCF) y tocodinamómetro externo para el registro de los movimientos fetales y la actividad uterina.

Para la realización de la prueba sin stress (NST) se utiliza la técnica descrita por Schifrin y colaboradores (10), de la siguiente manera:

Paciente en reposo, posición semisentada, toma periódica de lå̀ tensión arterial.

Localización del foco de la FCF y colocación del transductor de ultrasonido y el tocodinamómetro.

Registro durante un período mínimo de 20 minutos y máximo de 40 minutos. $\mathrm{Si}$ en los primeros 20 minutos no se obtienen movimientos fetales espontáneos o ascensos de la FCF, se practica estimulación manual al feto y se continúa la prueba por un período de 20 minutos adicionales.

En la interpretación del registro se toman en cuenta la línea de base, la variabilidad y las aceleraciones espontáneas.

Para el presente análisis se consideró como Reactiva la prueba cuando la línea de base estaba entre 120 y 160 latidos por minuto y había buena variabilidad de 5 a 20 latidos por minuto y se presentaban tres o más aceleraciones en 20 minutos, con una duración mínima de 15 segundos y un aumento en la frecuencia cardíaca fetal de 15 latidos por minuto o más.

La prueba se consideró No Reactiva cuando no cumplia estos requisitos.

A las pacientes con pruebas Reactivas se les citó ocho días después para repetirla.

Cuando la prueba se interpretó como No Reactiva, se procedió a practicar la prueba de tolerancia a las contracciones (PTC), utilizando la técnica descrita, por Ray y Colaboradores (8), en la siguiente forma:

Se inicia el registro en la misma forma descrita para la prueba sin stress (NST). Se aplica una infusión IV de 500 cc de Dextrosa en AD con 2 unidades de Syntocinon, se inicia el goteo muy lento con el fin de obtener 3 contracciones en un período de 10 minutos.

El criterio para considerar como Positiva la PTC, fue el mismo utilizado por Ray y colaboradores:

1. Desaceleraciones uniformes de la FCF.

2. Desaceleraciones que reflejen la contracción uterina.

3. Con un comienzo en el acmé o inmediatamente después de la contracción.

4. Que sea repetitiva con todas las contracciones.

La PTC se considera Negativa o Normal cuando no se presentan desaceleraciones.

Se considera Equívoca cuando se producen desaceleraciones que no cumplan los requisitos mencionados, o se producen más de 3 contracciones en los 10 minutos. 


\section{Resultados}

A las 162 pacientes se les practicó un total de 205 pruebas de NST y 54 de PTC, para un total de 259 pruebas realizadas.

Del total de pacientes estudiadas se encontró que el $55 \%$ (N: 89) correspondió a trastornos hipertensivos. (Tabla No. 1). En orden de frecuencia le siguieron post-madurez, antecedentes de muertes neonatales, diabetes, y otras causas.

Tabla No. 1

\section{INDICACIONES PARA}

PRUEBA DE N.S.T.

\begin{tabular}{lcr}
\hline \multicolumn{1}{c}{ Indicación } & No. Pacientes & \multicolumn{1}{c}{$\%$} \\
\hline Desórdenes hipertensivos & 89 & 55.0 \\
Post-madurez & 20 & 12.3 \\
Diabetes Mellitus & 14 & 8.6 \\
Antecedente de mortinatos & 14 & 8.6 \\
Incompatibilidad Rh - Sensib. & 13 & 8.0 \\
Primigestante añosa & 2 & 1.3 \\
Antecedente muertes neonatales & 3 & 1.8 \\
Cardiopatía & 1 & 0.6 \\
Placenta previa & 2 & 1.3 \\
Epilepsia & 1 & 0.6 \\
Polihidramnios & 2 & 1.3 \\
Incompat. Rh - No sensib. & -1 & 0.6 \\
\hline \multicolumn{1}{c}{ Total } & 162 & 100.0 \\
\hline
\end{tabular}

En la Tabla No. 2 se presentan los resultados de las 205 pruebas de NST. donde podemos ver que el $71.2 \%$ (N: 146) fueron Reactivas y el $28.8 \%$, (N: 59) fueron no Reactivas.

De las pruebas no reactivas, el $60 \%$ (N: 35) correspondieron a pacientes con desórdenes hipertensivos.

En la Tabla No. 3 podemos ver los resultados de las pruebas de tolerancia a las contracciones, realizadas en las pacientes con prueba de NST no reactiva.
Tabla No. 2

RESULTADO DE LAS

PRUEBAS DE N.S.T.

\begin{tabular}{lcc}
\hline \multicolumn{1}{c}{ Diagnóstico } & Reactivas & No. Reactivas \\
\hline Pre- eclampsia leve & 31 & 11 \\
Pre-eclampsia grave & 28 & 14 \\
Enf. V.H. Pre-eclam . leve & 2 & 7 \\
Enf. V.H. Pre-eclam.. grave & 6 & - \\
Enf. V.H. & 9 & 3 \\
Post-madurez & 12 & 10 \\
Diabetes mellitus & 14 & 7 \\
Iso-inmunización Rh & 15 & 5 \\
Antecedente muertes neonatales & 17 & - \\
Premigestante añosa & 5 & 2 \\
Cardiopatía & 1 & - \\
Placenta previa & 2 & - \\
Epilepsia & 1 & - \\
Polihidramnios & 2 & - \\
Incompatibilidad Rh & 1 & - \\
\hline \multicolumn{1}{c}{ Total } & $146(71.2 \%)$ & $59(28.7 \%)$ \\
\hline
\end{tabular}

De las 59 pacientes con prueba no reactiva, sólo se realizaron 54 pruebas de tolerancia a las contracciones. (PTC).

Tabla No. 3

\section{RESULTADO DE LAS PRUEBAS DE TOLERANCIA A LAS CONTRACCIONES}

\begin{tabular}{lccc}
\multicolumn{1}{c}{ Diagnóstico } & Positiva & Negativa & Equívoca \\
\hline Pre-eclampsia leve & 2 & 6 & - \\
Pre-eclampsia grave & 1 & 12 & 1 \\
Enf. V.H. Pre-eclam. leve & 3 & 6 & 1 \\
Enf. V.H. & 1 & 1 & - \\
Post-madurez & - & 8 & - \\
Diabetes mellitus & 1 & 4 & - \\
Iso-inmunización Rh & - & 5 & - \\
Primigestante añosa & 1 & 1 & - \\
\hline \multicolumn{1}{c}{ Total } & 9 & 43 & 2 \\
& $(16.6 \%)$ & $(79.6 \%)$ & $(3.8 \%)$ \\
\hline
\end{tabular}

En la Tabla No. 3 podemos observar que el $79.6 \%(\mathrm{~N}$ : 43) correspondió a PTC con resultados negativos y sólo en el $16.6 \% \quad(\mathrm{~N}: 9)$ correspondió a PTC 
con resultados positivos. Se encontró $3.8 \%(\mathrm{~N}: 2)$ de PTC equívocas.

De las 9 pacientes con PTC positivas el $66.6 \%$ (N: 7) correspondió a pacientes que presentaban desórdenes hipertensivos.

Tabla No. 4

\section{CORRELACION DE LA PRUEBA \\ DE TOLERANCIA A LAS \\ CONTRACCIONES P.T.C. CON NACIDOS VIVOS $Y$ MUERTES PERINATALES}

\begin{tabular}{lccr}
\hline Resultado & Muertós & Vivos & Total \\
\hline Positiva & 2 & 7 & 9 \\
Negativa & 1 & 44 & 45 \\
\hline Total & 3 & 51 & 54 \\
\hline
\end{tabular}

En la Tabla No. 4 se correlacionan los resultados de la PTC con los nacidos vivos $y$ las muertes neonatales. Es importante anotar que de 9 pacientes con PTC positiva hubo 2 muertes neonatales, mientras que de 43 pacientes con PTC negativa hubo una sola muerte neonatal.
En la Tabla No. 5 se presentan los casos de mortalidad. Aquí podemos observar que hubo un caso de muerte neonatal precedida de PTC negativa realizada ocho días antes del parto. Esta muerte ocurrió tres días posteriores al nacimiento. Se trataba de un caso de isoinmunización $\mathrm{Rh}$ con curva espectofotométrica III B (Lilly), para una edad gestacional de 34 semanas.

Los dos casos de nacidos muertos ocurrieron en pacientes con prueba de NST no reactiva y PTC positiva. Estas dos pacientes presentaban desórdenes hipertensivos.

En la Tabla No. 6, se presentan los resultados de las diferentes pruebas efectuadas, los promedios de edad gestacional en semanas al final del embarazo, peso del recién nacido e índice de Apgar al minuto y a los 10 minutos.

Como puede apreciarse, en la tabla no hay diferencia en cuanto a los promedios de los resultados entre las pruebas de NST reactiva y las pruebas de tolerancia a las contracciones (PTC) negativa.

En las pacientes con PTC positiva y su relación con la edad gestacional, es explicable que se encuentre edad gesta-

Tabla No. 5

RESUMEN DE LAS MUERTES PERINATALES

\begin{tabular}{|c|c|c|c|c|c|}
\hline Diagnóstico & NST/PTC & Edad Gestac. & Parto & Apgar & Observaciones \\
\hline E.V.H. P-ecl. leve & NR/POS & 32 Sem. & Vaginal & 0 & $\begin{array}{l}\text { Muerte anteparto } \\
\text { peso } 1.280 \text { gmos. }\end{array}$ \\
\hline E.V.H. P-ecl. leve & NR/POS & 33 Sem. & Vaginal & 0 & $\begin{array}{l}\text { Muerte anteparto } \\
\text { peso } 1.300 \text { gmos. }\end{array}$ \\
\hline Isoinmunización Rh & R/NEG. & 34 Sem. & Cesárea & $1 / 2$ & $\begin{array}{l}\text { Curva III B } \\
\text { Muerte neonatal } \\
\text { al tercer día } \\
\text { peso } 1.660 \text { gmos. }\end{array}$ \\
\hline
\end{tabular}


Tabla No. 6

CORRELACION ENTRE EL

RESULTADO DE LAS PRUEBAS

$Y$ LOS PROMEDIOS DE SEMANAS

DE GESTACION, PESO E INDICE DE APGAR

\begin{tabular}{lrccl}
\hline \multicolumn{1}{c}{ Prueba } & No. Ptes. & $\begin{array}{l}\text { Semanas } \\
\text { Gestac. }\end{array}$ & Peso & $\begin{array}{c}\text { Ind. de } \\
\text { Apgar }\end{array}$ \\
\hline NST Reactivas & 110 & 39.8 & 3.180 gmos & $8.7-9.6$ \\
PTC Negativas & 43 & 39.6 & 3.085 gmos & $7.8-9.5\left(^{*}\right)$ \\
PTC Positivas & 9 & 37.0 & 2.205 gmos & $5.5-7.7(-)$ \\
\hline
\end{tabular}

$\left({ }^{*}\right)$ Una muerte neonatal

(-) Dos muertes anteparto

cional más temprana, 37 semanas, ya que es necesario adelantar el parto.

Con respecto al peso, se encuentra que en las pacientes con PTC positiva el promedio (2.205 grs) es bajo para el E.G.

Con relación al Apgar, se ve una tendencia a índice más bajo (5.5-7.7), en el grupo de PTC positiva, mientras que en el grupo con PTC negativa los índices son muy similares a los observados en el grupo de pacientes con prueba de NST reactiva.

Tabla No. 7

COMPARACION CON OTROS ESTUDIOS SOBRE EL RESULTADO DE LAS PRUEBAS ANTEPARTO (PRUEBAS DE NST Y PTC)

\begin{tabular}{lrcc}
\hline Autor & $\begin{array}{r}\text { \% NST } \\
\text { Reactiva }\end{array}$ & $\begin{array}{c}\text { \% PTC } \\
\text { Negativa }\end{array}$ & $\begin{array}{c}\text { \% PTC } \\
\text { Positiva }\end{array}$ \\
\hline Presente estudio & 71.2 & 79.6 & $5.5(16.6)^{*}$ \\
Keane y Col. & 84.2 & 94.1 & 3.9 \\
Gibbons y Nagle & 90.2 & 63.0 & 37.0 \\
Paul y miller & 63.8 & 91.0 & 3.0 \\
\hline
\end{tabular}

* $5.5 \%$ del total de ptes. estudiadas (162) $16.6 \%$ del total de PTC.

\section{Comentarios y conclusiones}

Entre las ventajas del empleo de la monitoría fetal anteparto podemos mencionar que se trata de procedimiento no invasivo, de fácil aplicación e interpretación. Aunque se basa en criterios muy estrictos, estos no son difíciles de aprender.

La mayoría de los autores aceptan (3) (4) (6) (9) (10) el valor predictivo de buen pronóstico para el feto dentro de la semana siguiente a la ejecución de las pruebas, cuando la prueba de NST es reactiva y la PTC es negativa.

Por el contrario la prueba de NST no reactiva sin el complemento de la prueba de la tolerancia a las contracciones no tiene ningún valor pronóstico, ya que en condiciones fisiológicas como son el sueño, prematurez y la administración de drogas sedantes a la madre, pueden producir pruebas de NST no reactivas, sin que esto quiera decir que el feto se encuentre en malas condiciones.

En el presente estudio el $55 \%$ (N: 89) de las pacientes presentaban desordenes hipertensivos y por esta razón recibían sedación, principalmente fenobarbital, lo cual explicaría en parte el alto número de pruebas no reactivas seguidas de PTC negativas.

Esta diferencia, al compararla con otros estudios, puede deberse a la diferente patología materna que indicaba el empleo de la monitoría anteparto; como ejemplo vemos el predominio de la patología hipertensiva en nuestro trabajo, al contrario de otros trabajos en los que la post-madurez y la diabetes eran más frecuentes que los trastornos hipertensivos (3) (4) (5) (7) (11).

Comparando nuestro trabajo con el Keane y Col. (5) vemos que reportan $84 \%$ de pruebas de NST reactivas, pero 
sólo $26.5 \%$ de pacientes con trastornos hipertensivos.

En este trabajo se reportaron PTC negativas en el $94.1 \%$ y PTC positivas en el $3.9 \%$.

Otros autores han reportado entre $27 \%$ y $36 \%$ de pruebas de NST reactiva, (4) (5) (7).

La correlación entre pruebas de NST reactiva y PTC negativa ha sido demostrada por otros autores (4) (5) (7) (10).

La PTC es indispensable en última instancia para seleccionar cuáles son los fetos que están en peligro; ninguna prueba de NST no reactiva tiene valor predictivo si no está complementada con una PTC; esto no quiere decir que se justifique de rutina hacer PTC, como método aislado de bienesta ${ }^{*}$ fetal por las siguientes razones:

1. Ya ha sido demostrada por muchos autores la correlación entre las dos pruebas (5) (7) (10).

2. La prueba de NST no tiene ninguna contraindicación ni representa ninguna molestia para la paciente.

3. Toda prueba reactiva indica que el feto está bien y toda prueba no reactiva es indicación de PTC la cual en última instancia es la que va a dar valor predictivo sobre el estado fetal.

En el presente trabajo únicamente el $5.5 \%$ presentó PTC positiva y precisamente en este grupo encontramos dos de las muertes perinatales ocurridas.

En cuanto a la otra muerte neonatal, había pruebas de NST/PTC normales 8 días antes del parto y por lo tanto de acuerdo a los criterios establecidos no podríamos clasificarla como falsa negativa.
Con relación al $79.6 \%$ de PTC negativa en nuestro trabajo, se explica por la misma razón de tener NST no reactiva, por considerar que estos fetos han estado bajo efecto de drogas, pero su reserva respiratoria no ha sido comprometida $y$ por lo tanto toleran bien las contracciones.

En la Tabla No. 7 se presenta la comparación del presente trabajo con otros estudios sobre el resultado final de las pruebas de NST y PTC.

Vemos como nuestros resultados están dentro de los límites reportados por estos autores (5) (4) (7).

Como ya se dijo, existe controversia entre hacer o no hacer PTC precedida de prueba de NST reactiva; este estudio confirma lo demostrado por otros autores: que pruebas de NST reactiva son indicativas de bienestar fetal y por lo tanto no es necesario complementarlas con PTC, (5) (6).

\section{Resumen}

Se presenta el resultado de 259 pruebas, 205 pruebas de NST y 54 de PTC, efectuadas en 162 pacientes clasificadas como de alto riesgo en el Departamento de Gineco-obstetricia del Hospital SanIgnacio.

La patología preponderante fueron los desórdenes hipertensivos 55\% (N: 89). El $71.2 \%$ (N: 146) de las pruebas de NST fueron reactivas y el $28.8 \%$ (N: . 59) fueron no reactivas; de estas 54 se les practicó PTC: 79.6 (N: 43) fueron negativas, el $16.6 \%$ ( $\mathrm{N}: 9)$ fueron positivas y el $3.8 \%$ (2) fueron equívocas.

Hubo tres muertes perinatales: dos ocurrieron en pacientes que tenían PTC positiva y en la otra aunque la PTC fue negativa, había transcurrido más de una 
semana entre la ejecución de la prueba y el parto.

Se relaciona el resultado final del embarazo (peso, semanas de gestación y Apgar) con las pruebas empleadas, encontrándose que existe una correlación entre la PTC positiva y la mortalidad perinatal, el peso más bajo al nacimiento, el índice de Apgar más bajo y el posible retardo del crecimiento intrauterino en estas pacientes.

Se confirma la utilidad pronóstica de la monitoría fetal anteparto en pacientes de alto riesgo.

\section{ANTEPARTUM FETAL MONITORING AT THE HIGH RISK UNIT SAN IGNACIO HOSPITAL}

\section{Summary}

This article presents the results of 259 tests, of which 205 were NST and 54 were PTC, performed on 162 high risk patients at the Department of Obstetrics and Gynecology of San Ignacio Hospital.

The predominant pathology was hypertensive disorders: $55 \%$ (N: 89). $71.2 \%$ (N: 146) of the NST tests were reactive, while $28.8 \%$ (N: 59) were non-reactive. Out of 54 PTC tests, $79.6 \%$ (N: 43) were negative, $16.6 \%$ $(\mathrm{N}: 9)$ were positive, and $3.8 \%(\mathrm{~N}: 2)$ were uncertain.

\section{Bibliografía}

1. CARRERA JM. MALLAFRE J. ALE. GRE M. PETRACC A. TAMAYO; Evaluación anteparto de la reserva respiratoria fetoplacentaria. Monitorización fetal anteparto. Barcelona. Edit. Salvat. 1a Edición. 1980.

2. CHAN YL. FACOG P. DILORETO: Fetal activity acceleration determination for the evaluation of fetal reserve. Obstet. Gynecol. 48: 19. 1976.
There were three perinatal deaths: two of those women had had positive PTC, and for the other one, in spite of the fact that PTC was negative, it was performed more than a week before delivery.

Through the tests, there seems to be a close relationship between the results of the gestation (weight, weeks of gestation and Apgar) and the test, with a correlation between a positive PTC and perinatal mortality, lower birth weight, lower Apgar index, and possible retardation of uterine growth in these patients.

Authors confirm the usefulness of antepartum fetal monitoring in high risk patients.

3. CHIN CHUN LIN et al: An OCT. Reactivity Classification to predict fetal outcome. Obstet Gynecol. 56:17 1980.

4. GIBBONS J. NAGLE P; Correlation of Nonstressed fetal heart rate with sequential contraction stress test. Obstet Gynecol, 55:612. 1980.

5. KEANE M. et al: Comparative study of stressed and nonstressed antepartum fetal heart rate, testing, Obstet Gynecol 57: 320. 1981. 
6. PAUL RH, hON EH: Clinical fetalmonitoring. $V$. Effect on perinatal outcome. Am J Obstet Gynecol 118:529. 1974.

7. PAUL RH, MILLER: Antepartum fetal heart monitorn. Clin Obstet Gynecol. 21:375, 1978.

8. RAY M. FREEMAN R. PINE S. HESSELGESSEN R: Clinical experiencie with the exytocin challenge test. Am J. Obstet. Gynecol. 114:1; 1972.
9. ROCHARD F. SCHIFRIN B. GOUPIL F. LEGRAND H. SUREAU C: Nostressed fetal heart rate monitorin in the antepartum period. Am J. Obstet Gyne col, 126: 699. 1976.

10. SCHIFRIN B et al: Routine fetal heart rate monitoring in the antepartum period Obstet Gynecol. 54:21, 1979.

11. SCHIFRIN B: Contraction stress test for antepartum fetal evaluation. Obstet Gynecol 45:433. 1975. 